\title{
Probabilidade de chuvas em Bom Jesus - Piauí, Brasil
}

\author{
Rain probability in Bom Jesus - Piauí, Brazil \\ Probabilidad de lluvia en Bom Jesus - Piauí, Brasil
}

Recebido: 05/02/2022 | Revisado: 11/02/2022 | Aceito: 16/02/2022 | Publicado: 24/02/2022

\author{
Luciano Marcelo Fallé Saboya \\ ORCID: https://orcid.org/0000-0002-7586-6867 \\ Universidade Federal de Campina Grande, Brasil \\ E-mail: 1saboya@hotmail.com \\ Raimundo Mainar de Medeiros \\ ORCID: https://orcid.org/0000-0001-7361-1281 \\ Universidade Federal Rural de Pernambuco, Brasil \\ E-mail: mainarmedeiros@gmail.com \\ Romildo Morant de Holanda \\ ORCID: https://orcid.org/0000-0001-7945-3616 \\ Universidade Federal Rural de Pernambuco, Brasil \\ E-mail: romildomorant@gmail.com \\ Manoel Viera de França \\ ORCID: https://orcid.org/0000-0003-4973-9327 \\ Universidade Federal Rural de Pernambuco, Brasil \\ E-mail: manoelvieira.ufrpe@gmail.com \\ Wagner Rodolfo de Araújo \\ ORCID: https://orcid.org/0000-0001-7203-0338 \\ Universidade Estácio de Sá, Brasil \\ E-mail: wagneraraujops@gmail.com \\ Moacyr Cunha Filho \\ ORCID: https://orcid.org/0000-0002-3466-8143 \\ Universidade Federal Rural de Pernambuco, Brasil \\ E-mail: Moacyr.cunhafo@ufrpe.br
}

\begin{abstract}
Resumo
Os estudos de probabilidade de ocorrência de chuvas são importantes em razão do adequado dimensionamento de obras públicas, como sistemas de drenagem, e de práticas de manejo e conservação do solo, que devem ser implementados pelos agricultores. Objetiva-se estudar a probabilidade de episódios de chuvas utilizando dados de precipitações mensais e anuais, para o munícipio de Bom Jesus. Trabalhou-se com o período de 1960 a 2018, os quais foram analisados quanto a consistência, homogeneização e preenchimentos de falhas e ordenação. Obteve-se informações de precipitação diária, mensal e anual, gerando informações sobre valores máximos absolutos diários e mensais; máxima, média e mínima mensal e anual. Ajustou-se a precipitação mensal a distribuição gama. A probabilidade de ocorrência de chuvas no município de Inconfidentes é variável para cada mês, mas segue um padrão no decorrer dos anos. Embora meses como janeiro, fevereiro e outubro, sejam mais críticos com a probabilidade de $4,4 \%, 2,2 \%$ e $6,7 \%$, respectivamente para ocorrência de chuvas com mais de $90 \mathrm{~mm}$ diários e menor incidência nos meses de junho e julho com probabilidade de aproximadamente $0 \%$. A possibilidade de anomalias na precipitação é real mesmo que com a baixa probabilidade. Determinando a disponibilidade hídrica, a probabilidade de ocorrência de eventos extremos e o período de retorno pode-se fazer um planejamento otimizado, ajudando no desenvolvimento de vários tipos de projetos, trazendo benefícios para a sociedade local.
\end{abstract}

Palavras-chave: Precipitação; Probabilidade; Distribuição Gama.

\begin{abstract}
Rainfall probability studies are important for the adequate design of public works, such as drainage systems, and for soil management and conservation practices that should be implemented by farmers. The objective of this study was to study the probability of rainfall episodes using monthly and annual precipitation data for the municipality of Bom Jesus. We worked with the period from 1960 to 2018, which were analyzed for consistency, homogenization and filling of gaps and ordering. Daily, monthly, and annual precipitation information was obtained, generating information about daily and monthly absolute maximum values; monthly and annual maximum, average, and minimum values. The monthly precipitation was fitted to the gamma distribution. The probability of rainfall occurrence in the municipality of Inconfidentes is variable for each month, but follows a pattern over the years. Although months like January, February and October, are more critical with a probability of $4.4 \%, 2.2 \%$ and $6.7 \%$, respectively for the occurrence of rainfall of more than $90 \mathrm{~mm}$ daily and a lower incidence in the months of June and July with a probability of approximately $0 \%$. The possibility of precipitation anomalies is real even with the low
\end{abstract}


probability. Determining the availability of water, the probability of occurrence of extreme events and the return period can make an optimized planning, helping in the development of various types of projects, bringing benefits to the local society.

Keywords: Precipitation; Probability; Gamma Distribution.

\section{Resumen}

Los estudios de probabilidad de lluvia son importantes para el diseño adecuado de las obras públicas, como los sistemas de drenaje, y para las prácticas de gestión y conservación del suelo que deben aplicar los agricultores. El objetivo fue estudiar la probabilidad de los episodios de precipitación utilizando datos de precipitación mensual y anual para el municipio de Bom Jesus. Se trabajó con el período comprendido entre 1960 y 2018 , que fueron analizados para su consistencia, homogeneización y llenado de vacíos y ordenamiento. Se obtuvo información de las precipitaciones diarias, mensuales y anuales, generando información sobre los valores máximos absolutos diarios y mensuales; valores máximos, medios y mínimos mensuales y anuales. La precipitación mensual se ajustó a la distribución gamma. La probabilidad de ocurrencia de lluvias en el municipio de Inconfidentes es variable para cada mes, pero sigue un patrón a lo largo de los años. Aunque meses como enero, febrero y octubre, son más críticos con una probabilidad del 4,4\%, 2,2\% y 6,7\%, respectivamente para la ocurrencia de precipitaciones de más de $90 \mathrm{~mm}$ diarios y menor incidencia en los meses de junio y julio con probabilidad de aproximadamente $0 \%$. La posibilidad de que se produzcan anomalías en las precipitaciones es real, aunque con baja probabilidad. La determinación de la disponibilidad de agua, la probabilidad de ocurrencia de eventos extremos y el período de retorno puede hacer una planificación optimizada, ayudando en el desarrollo de varios tipos de proyectos, trayendo beneficios a la sociedad local.

Palabras clave: Precipitación; Probabilidad; Distribución Gamma.

\section{Introdução}

Segundo Marengo (2009), eventos climáticos extremos ocorrem de muitas maneiras e formas, tais como: enchentes, alagamentos, cheias, inundações, deslizamentos de terra, secas prolongadas, redemoinhos e ondas de calor. Estes eventos vêm mudando e a partir da segunda metade do século XX, observou-se que devido à ação antrópica essas ocorrências vêm se intensificando. O excesso e a escassez pluvial podem ocasionar eventos adversos impactando diferentemente os setores econômicos, a nível local, estadual e federal (Alvarenga, 2012).

A estimativa da probabilidade de episódios de chuvas extremas, com a devida antecedência, podem proporcionar ações mitigadoras antecipadas, por parte dos órgãos executivos da administração pública, e dos empreendimentos privados, no intuito de diminuição de danos à sociedade e ao meio ambiente (Junqueira Júnior et al., 2015).

Para a efetivação de planejamento e desenvolvimento urbanos, agrícolas e hidrológicos, entre tantos outros projetos, as estimativas probabilísticas das variáveis meteorológicas são fundamentais e podem ser usadas para estimar eventos raros e/ou extremos, que possuem grande relevância climatológica, hidrológica e socioeconômica (Sansigolo, 2008).

Os efeitos devastadores dos eventos climáticos sobre a população da zona urbana ou mesmo rural, proporcionados pelas enchentes, alagamento, cheias, desmoronamento de ribanceiras, ocorrem devido ao pouco ou a não existência de planejamento, associada ao uso inadequado das áreas (Brito et al., 2015), os quais poderiam ser evitados e/ou corrigidos com o conhecimento dos elementos meteorológicos históricos e com o planejamento urbano/rural. A estimativa da probabilidade de ocorrência de precipitações diárias máximas anuais podem ser locais, ou seja, utilizando-se a série de dados de uma única estação, ou regional, fazendo uso dos dados de várias estações de uma área (Weschenfelder et al. 2011).

Saboya et al, (2021), Os estudos sobre a distribuição da precipitação favorecem a colocação da quantidade de água necessária para o desenvolvimento das atividades humanas, sejam elas pessoais, industriais ou agrícolas. O objetivo deste trabalho é modelar estatisticamente os valores médios mensais da precipitação e estimar a respectiva precipitação em diferentes níveis de probabilidade, através da distribuição Gama, para o Município de Barbalha - Ceará, Brasil, no período de 1973 a 2017. O Modelo da Função Probabilística Gama e os dados mensais do teste de aderência foram avaliados pelo teste Kolmogorov-Smirnov. Os meios variam de 3,6 mm (Agosto) a 237,4 mm (Março). Os quatro meses com maior pluviosidade são registados entre Janeiro e Abril, e o que tem os índices mais baixos ocorre entre Junho e Setembro. A oscilação do 
parâmetro alfa ocorreu de 0,829 (Agosto/Setembro) a 0,896 (Março); o parâmetro beta oscilou entre 0,851 (Agosto/Setembro) a 0,922 (Março). Quanto à probabilidade empírica de ocorrência de precipitação anual total, a percentagem destaca-se $50 \%$ e $70 \%$ com possibilidades de chuva superiores à média histórica. As oscilações positivas da anomalia fluíram entre $75 \mathrm{~mm}$ a 910 $\mathrm{mm}$. As anomalias negativas oscilaram entre 55,5 mm em 2005 e $432 \mathrm{~mm}$ em 1982.

O índice pluvial é a principal componente do ciclo hidrológico, pois representa a maior entrada de água nas bacias hidrográficas. Quando ocorre o aumento destes índices registra-se uma situação crítica, denominada de eventos extremos. Sendo que, um evento pluvial intenso é considerado, quando apresenta valores superiores ao percentil de 95\% de uma série de dados, enquanto, o evento pluviométrico extremo, é considerado para valores superiores ao percentil de $99 \%$. A análise dos impactos gerados pelas chuvas extremas é de suma importância para sociedade, pois determina áreas de riscos para as habitações e atividades agropecuárias. É de suma importância para o planejamento de obras hidráulicas de controle de cheias e inundações, para a drenagem urbana e na adoção de práticas de manejo e conservação do solo na zona rural, represamento e abastecimento de água urbano e/ou rural. (Mello et al., 2007; Mello et al., 2013)

As oscilações climáticas podem ser compreendidas como uma característica natural do sistema climático terrestre, responsável por variabilidades naturais dos padrões climáticos, previstos em nível local, regional e global (Confalonieri, 2003). No caso do Brasil, a disponibilidade de água depende em grande parte do clima, sendo que o ciclo anual das chuvas e de vazões nas bacias hidrográficas varia significativamente dentro de uma mesma região ou local, portanto a variabilidade interanual do clima, associada aos fenômenos climáticos de larga escala, como o El Niño(a) podem ocasionar anomalias climáticas, os quais podem ocasionar grandes secas ou chuvas intensas (Marengo, 2008).

As anomalias climáticas são eventos meteorológicos e climatológicos que fogem dos valores médios, como exemplo a precipitação, que pode ser muito superior ao valor médio ou muito abaixo, gerando uma anomalia que pode causar desde enchentes até grandes períodos de estiagem (Pereira et al., 2007).

Os conhecimentos das distribuições dos regimes pluviais demonstrados no computo do balanço hídrico (BH), é de suma importante para um manejo conservacionista do solo e da água (Santo et al., 2009). Sendo de extrema necessidade o conhecimento das ocorrências dos eventos extremos, como precipitações máximas diárias e precipitações totais mensais, as quais não são apresentadas no balanço hídrico climatológico (BHC), mas que causam grandes dificuldades e/ou tragédias tanto em áreas urbanas e rurais.

Os estudos de Probabilidade de ocorrência de chuvas extremas são importantes para a área estudada, visto não só pela aptidão agrícola da região, mas os problemas de caráter urbano, como sistemas de drenagem, que podem ser mais bem projetados/dimensionados com base neste estudo, além do poder erosivo do qual vem ocorrendo no município.

Têm-se como objetivo estudar a probabilidade de episódio de chuvas extremas utilizando dados de precipitações mensais e anuais para o munícipio de Bom Jesus do Piauí no período de 1960 a 2018.

\section{Material e Método}

A área em estudo compreende o município de Bom Jesus - Piauí, com coordenadas geográficas de latitude $09^{\circ} 04^{\prime} \mathrm{S}$ e longitude $44^{\circ} 21^{\prime} \mathrm{W}$ e altitude de 220 metros (Figura 1). 
Figura 1. Localização do município dentro do Estado.

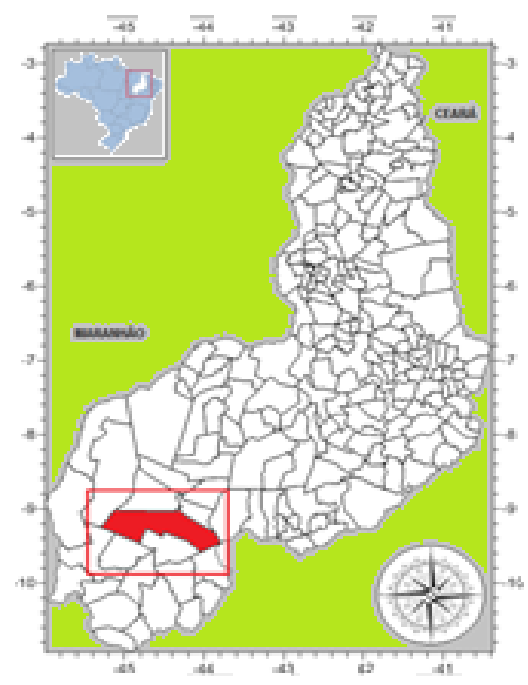

Fonte: Medeiros (2022).

De acordo com a classificação de Köppen (1928, 1931) o clima da área de estudo é do tipo Aw', tropical quente e úmido, com chuvas no verão e seca no inverno. As chuvas são formadas pelas penetrações dos vestígios das frentes frias, as contribuições das Zonas de Convergência do Atlântico Sul e os ventos alísios de sudeste, com precipitação média anual de 984,8 mm (Medeiros, 2018 e Alvares et al., 2014).

Dadas às informações climatológicas e dinâmicas do Nordeste do Brasil (NEB) e em especial para o município de Bom Jesus do Piauí tem seu clima controlado pela variabilidade espacial e temporal da Zona de Convergência do Atlântico Sul (ZCAS), e pelos vestígios das frentes frias, contribuição dos vórtices ciclônicos de atlântico sul (VCAS), desde que seu centro de atuação esteja no oceano, às formações e intensificações das linhas de instabilidade e dos aglomerados convectivos auxiliados pelos ventos alísios de sudeste, a convergência de umidade e a troca de calor sensível por latente e vice-versa. As contribuições dos efeitos locais, fatores que aumentam a cobertura de nuvens, a umidade relativa do ar e provocam chuvas de intensidades moderadas as fracas em quase todos os meses do ano, sendo o fenômeno La Niña o principal fator para ocorrência de chuvas acima da média histórica provocando inundações, alagamentos, enchentes, enxurradas e desmoronamento. (Figura 2) (Medeiros, 2016). 
Figura 2. Regimes e principais fatores provocadores de chuva no estado do Piauí.

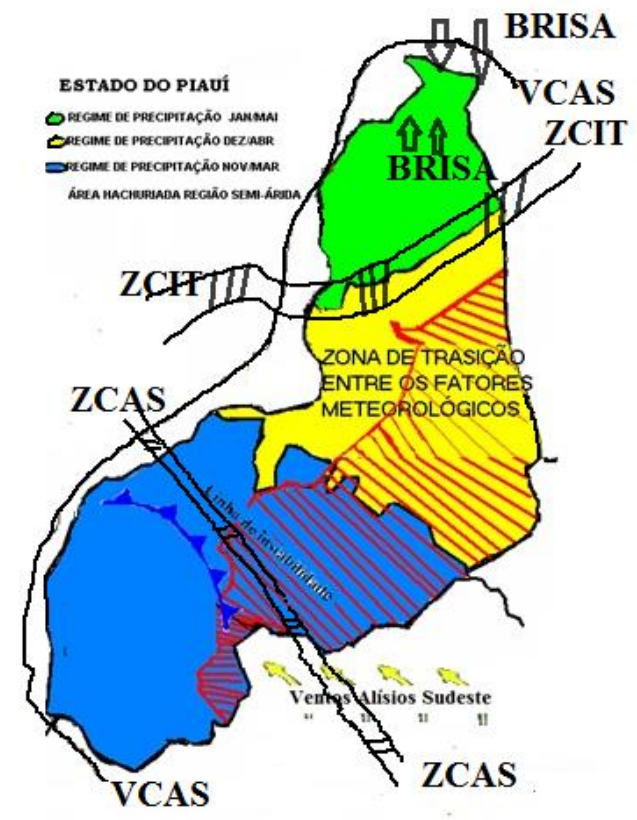

Fonte: Medeiros (2016).

Os dados obtidos corresponderam ao período de 1960 a 2018 adquiridos da Superintendência do Desenvolvimento do Nordeste (Sudene, 1990), Empresa de Extensão Rural do Estado do Piaú́ (Ematerpi, 2019) e do Instituto Nacional de Meteorologia (INMET, 2019), foram analisados quanto a consistência, homogeneização, realizado preenchimentos de falhas e ordenação. Obteve-se informações de precipitação diária, mensal e anual máxima, média e mínima e seus respectivos valores máximos absolutos diários e mensais, com o intuito de monitorar as variabilidades pluviais e observar anomalias, analisados através da divisão em 5 períodos de 12 anos.

Adotou-se o método simplificado para realizar o computo da ocorrência da probabilidade de chuvas a partir de uma sequência de valores medidos, baseando-se na ordenação crescente ou decrescente dos valores. Se a ordenação for crescente, a probabilidade corresponderá a um valor igual ou menor que o limite escolhido; se for decrescente, estima-se o inverso. Para obter um episódio de probabilidade de ocorrência de chuvas de um dado valor maior ou menor que um valor crítico, ordenouse a série de dados em ordem decrescente, respectivamente, e dividiu-se o número de ordem (m) correspondente à posição do valor crítico pelo número total de dados (n) mais 1, como mostrado na equação (Pereira et al., 2007):

$$
P=\frac{m}{n+1} x 100
$$

Onde:

P - Probabilidade de ocorrência em \%;

M - Número da ordem;

N - Número de dados total;

Caso a sequência de dados, registre ocorrência de valores nulos (ausência de chuva), eles devem ser descartados, anotando-se quantas vezes isto ocorreu (No), fazendo-se depois a ordenação dos valores restantes. Nesse caso, a probabilidade de ocorrência (P) de um valor crítico será dada pela equação (Pereira et al., 2007): 


$$
P=\left(1-\frac{N o}{n}\right) \times\left(\frac{m}{n+1-N o}\right) \times 100
$$

Onde:

P - Probabilidade de ocorrência em \%;

No - Número de valores nulos de precipitação;

n - Número de dados total;

m - Número da ordem

Ressalta-se que ao trabalhar com ocorrência de probabilidade de uma componente meteorológica é de fundamental importância obter-se a distribuição de probabilidade do período de retorno ou intervalo médio de recorrência (t). Em regra geral, calcula-se o período de retorno para valores extremos, ou seja, nos casos de anomalias climáticas que venham a causar impactos socioeconômicos, e não para aqueles valores que ocorrem normalmente. Para que a estimativa de $\mathrm{t}$ seja a melhor possível de ajuste, é coerente que se trabalhe com uma série longa de valores observados, pois t depende da probabilidade de ocorrência (P) do evento em estudo (Pereira et al., 2007).

Quanto mais frequente o valor crítico avaliado, menor será o período de retorno. Trabalhou-se com os dados da série em ordenação decrescente, utilizando-se da equação:

$$
t=\frac{1}{p}
$$

Onde:

$\mathrm{t}$ - Período de retorno, em anos;

P - Probabilidade de ocorrência, em decimal.

Com a obtenção dos valores de probabilidade e período de retorno adotou-se os índices pluviais de 50, 60, 70, 80 e 90 mm para valores diários e para o total mensal pluvial os valores de 25, 50, 150, 300 e 500 mm. Após as efetivações da probabilidade e do período de retorno representaram-se os referidos dados em Tabelas. Wilks (2006).

A estimativa dos índices pluviais mensais prováveis foi determinada para os níveis de 10, 20, 30, 40, 50, 60, 70, 75, 80, 90 e 95\% de probabilidade utilizando o modelo Gama incompleta (Thom, 1958). Os elementos de ajuste da função de distribuição Gama, alfa $(\alpha)$ e beta $(\beta)$, foram estimados pelo método da máxima verossimilhança, com a aproximação polinomial para o parâmetro $\alpha$ proposto por Greenwood e Durand (1960) apud Wilks (2006). Utilizou-se o teste KolmogorovSmirnov (KS) ao nível de significância de 5\% para determinar a adequabilidade do ajuste adotado em cada mês (Warrick et al., 1980).

\section{Resultados e Discussões}

O planejamento e o desenvolvimento de áreas urbanizadas e agricultáveis devem levar em consideração as estimativas probabilísticas dos índices pluviais básicos, para prever eventos infrequentes e/ou extremos, os quais possuem grandes relevâncias climatológica, hidrológica e socioeconômica. Nas Tabelas observam-se os valores em porcentagem de probabilidade de ocorrência de precipitações máxima e o tempo de período de retorno em anos com dados diários e mensais. Seguidamente dos valores de precipitações as máximas, médias e mínimas mensais do período de 1960-2019.

Na Figura 3 observa-se a variação da precipitação mensal (mm) para Bom Jesus do Piauí-PI, destaca-se o período chuvoso com início de outubro a abril, e o período seco de maio a setembro. 
Figura 3. Observa-se a variação da precipitação mensal.

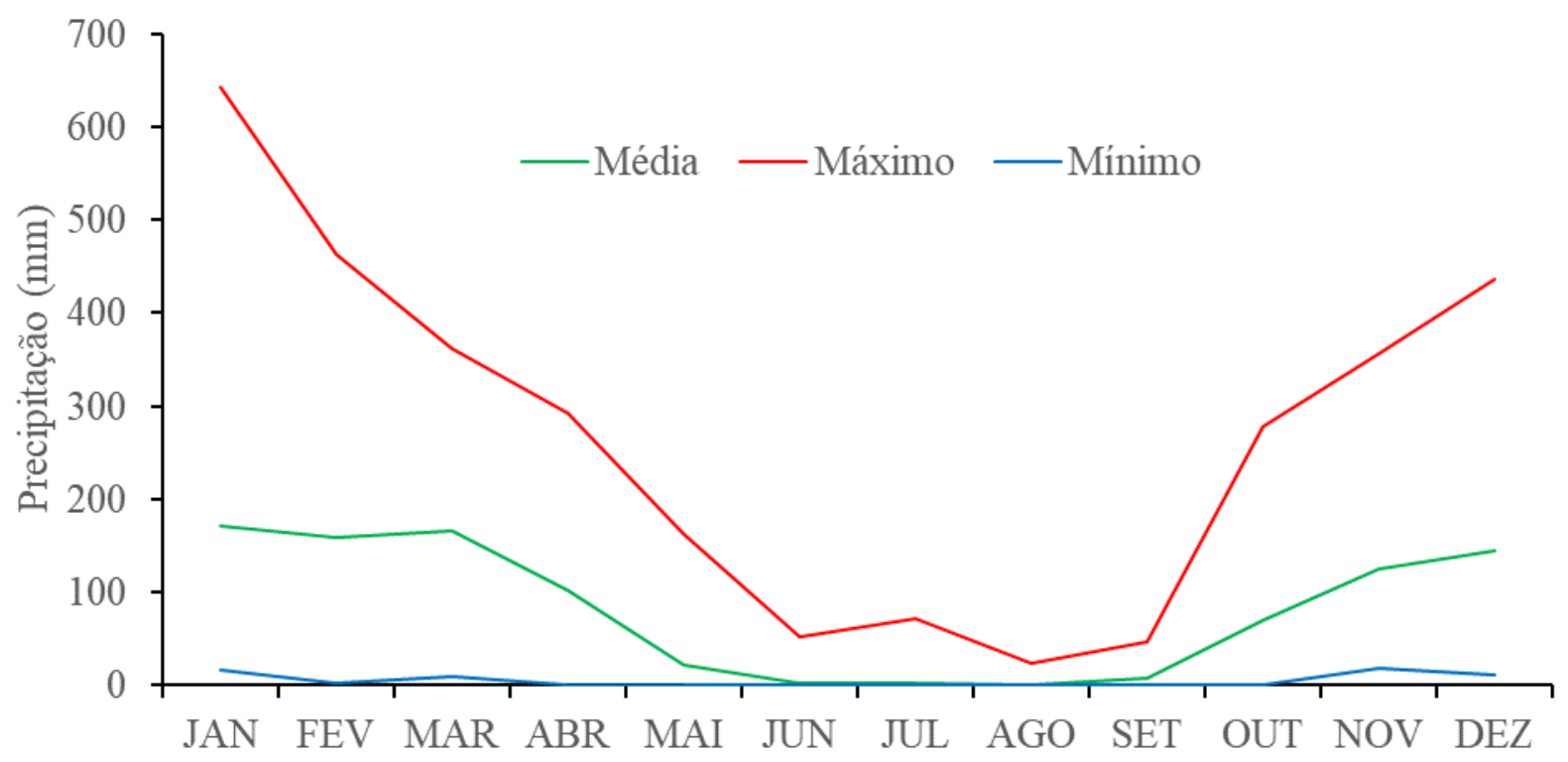

Fonte: Saboya (2022).

A precipitação média foi em outubro $(70,3 \mathrm{~mm})$, novembro $(124,6 \mathrm{~mm})$, dezembro $(144,7 \mathrm{~mm})$, janeiro $(171,7 \mathrm{~mm})$, fevereiro (158,6 mm), março $(166,4 \mathrm{~mm})$ e abril $(101,5 \mathrm{~mm})$, destacando-se a ocorrência de máxima para janeiro de $642,5 \mathrm{~mm}$ em 2004, e a mínima de 17,5 mm, neste mês, denotando a alta variabilidade da distribuição pluviométrica para o município. Este comportamento é característico do cerrado brasileiro, em que algumas ocorrências de veranicos são esperadas, mas tudo depende dos fatores climáticos reinantes.

Nos meses de maio $(22,6 \mathrm{~mm})$, junho $(3,3 \mathrm{~mm})$, julho $(2,3 \mathrm{~mm})$, agosto $(1,4 \mathrm{~mm})$ e setembro $(8,5 \mathrm{~mm})$ temos os referidos valores médios, sendo característicos do período seco, observa-se ausência de precipitações nos referidos meses, implicando em adoção de práticas agrícolas de convivência com escassez hídrica.

Com a divisão de 5 períodos de 12 anos cada, dos 48 anos estudados, notou-se que a precipitação segue um padrão no qual tem uma notável presença de janeiros e dezembros chuvosos, e junhos, julhos e agostos secos; definindo verões úmidos e invernos secos (Figura 4).

Analisando-se os 5 períodos comparativamente, não se observa tendências de elevação ou redução nas precipitações médias mensais, nem modificação no perfil de distribuição das chuvas ao longo dos anos estudados (Figura 4). 
Figura 4. Distribuições das precipitações médias mensais do período de 1960 a 2018, com representação de 5 períodos de 12 anos, para o município de Bom Jesus do Piauí.

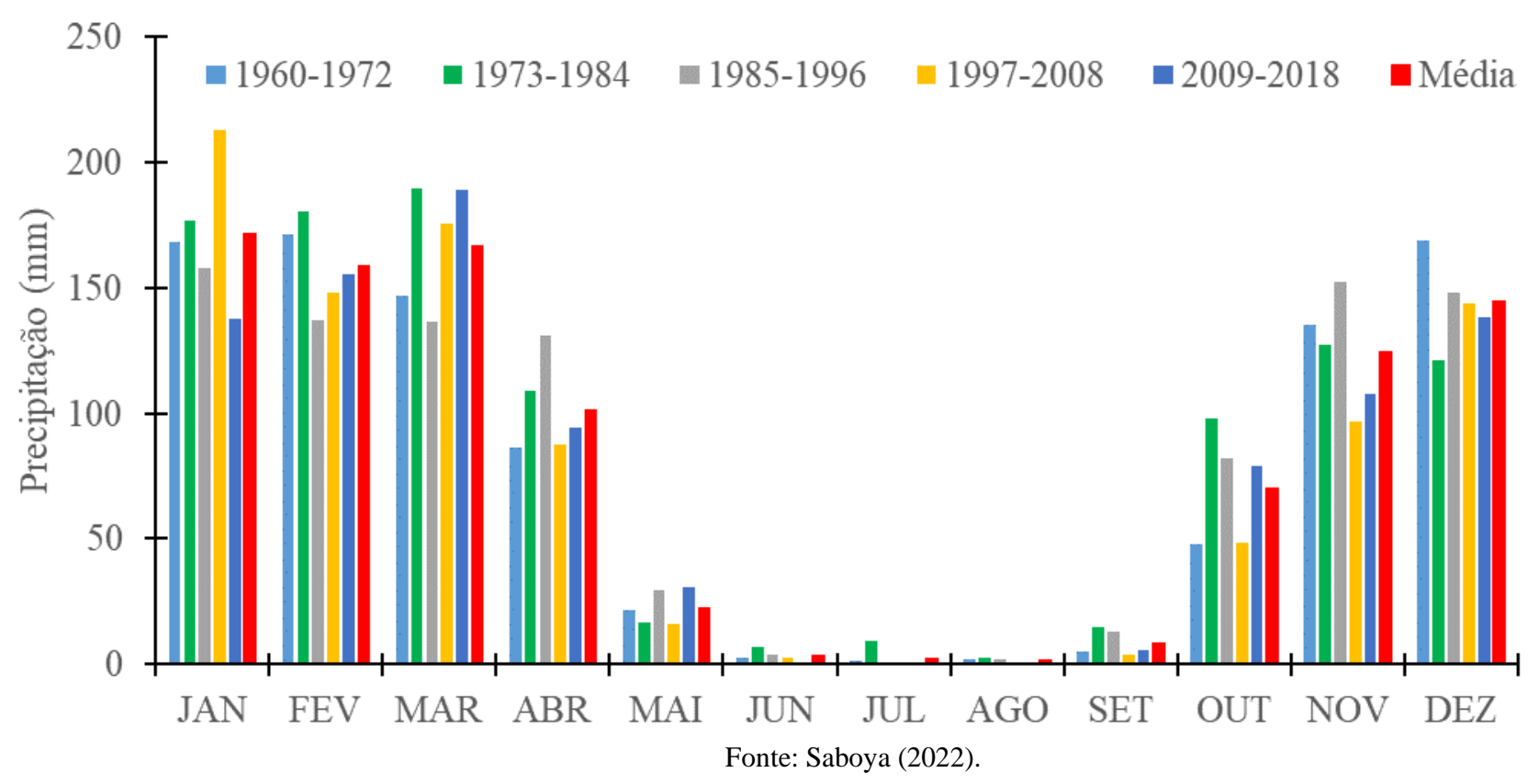

Na Tabela 1 observa-se os parâmetros de ajuste para distribuição de probabilidade gama, para cada mês, e os valores de precipitação esperados para diferentes níveis de probabilidade de ocorrência. Os ajustes para cada mês são considerados adequados pois os valores de D observados foram inferiores aos D calculado, quando da aplicação do teste de Kolmogoro. 
Tabela 1. Probabilidade de ocorrência (\%) de precipitação máxima mensal acima dos valores críticos estabelecidos.

\begin{tabular}{|c|c|c|c|c|c|c|c|c|c|c|c|c|c|}
\hline \multirow{2}{*}{\multicolumn{2}{|c|}{$\begin{array}{c}\text { Probabilidade } \\
(\%)\end{array}$}} & \multicolumn{12}{|c|}{ Meses } \\
\hline & & jan & fev & mar & abr & mai & jun & jul & ago & set & out & nov & dez \\
\hline \multicolumn{2}{|c|}{10} & 65,6 & 50,6 & 66,6 & 12,7 & 12,7 & 0,0 & 0,0 & 0,0 & 0,0 & 4,4 & 40,9 & 54,0 \\
\hline \multicolumn{2}{|c|}{20} & 90,2 & 73,8 & 90,2 & 25,0 & 25,0 & 0,0 & 0,0 & 0,0 & 0,2 & 11,1 & 59,1 & 74,8 \\
\hline \multicolumn{2}{|c|}{30} & 111,5 & 94,7 & 110,5 & 38,5 & 38,5 & 0,2 & 0,1 & 0,1 & 0,5 & 19,7 & 75,4 & 93,0 \\
\hline \multicolumn{2}{|c|}{40} & 132,4 & 115,6 & 130,2 & 53,6 & 53,6 & 0,4 & 0,2 & 0,2 & 1,3 & 30,3 & 91,6 & 110,7 \\
\hline \multicolumn{2}{|c|}{50} & 132,4 & 115,6 & 130,2 & 71,1 & 71,1 & 0,4 & 0,2 & 0,2 & 1,3 & 30,3 & 91,6 & 129,3 \\
\hline \multicolumn{2}{|c|}{60} & 178,1 & 162,7 & 173,1 & 92,2 & 92,2 & 1,7 & 1,0 & 0,8 & 4,6 & 60,3 & 128,0 & 149,9 \\
\hline \multicolumn{2}{|c|}{70} & 206,5 & 192,4 & 199,5 & 119,1 & 119,1 & 3,0 & 1,9 & 1,4 & 7,9 & 82,6 & 151,0 & 174,2 \\
\hline \multicolumn{2}{|c|}{75} & 223,4 & 210,4 & 215,2 & 136,0 & 136,0 & 4,0 & 2,7 & 1,8 & 10,3 & 97,1 & 164,8 & 188,7 \\
\hline \multicolumn{2}{|c|}{80} & 243,2 & 231,6 & 233,6 & 156,6 & 156,6 & 5,3 & 3,6 & 2,4 & 13,5 & 115,1 & 181,1 & 205,8 \\
\hline \multicolumn{2}{|c|}{$90 \%$} & 300,8 & 293,9 & 286,8 & 219,9 & 219,9 & 10,0 & 7,2 & 4,4 & 24,9 & 172,1 & 228,9 & 255,3 \\
\hline \multicolumn{2}{|c|}{$95 \%$} & 354,3 & 352,6 & 336,2 & 282,7 & 282,7 & 15,3 & 11,4 & 6,7 & 37,6 & 230,3 & 273,9 & 301,5 \\
\hline \multirow[b]{2}{*}{ Gama } & alfa & 3,2 & 2,5 & 3,4 & 1,12 & 0,4 & 0,3 & 0,3 & 0,3 & 0,3 & 0,8 & 2,6 & 3,1 \\
\hline & beta & 53,9 & 64,3 & 48,4 & 87,6 & 58,6 & 10,6 & 8,9 & 4,6 & 25,1 & 90,2 & 48,7 & 47,1 \\
\hline \multirow{2}{*}{$\begin{array}{c}\text { Teste } \\
\text { KS } \\
\text { a } 5 \%\end{array}$} & Dob & 0,022 & 0,035 & 0,010 & 0,126 & 0,105 & 0,024 & 0,066 & 0,045 & 0,053 & 0,109 & 0,029 & 0,038 \\
\hline & $D c$ & \multicolumn{12}{|c|}{0,17706} \\
\hline
\end{tabular}

Legenda: Dob= Valor observado de D; Dc= Valor crítico de D. Fonte: Saboya (2022).

Em relação a análise de probabilidade de ocorrência pode-se observar na Tabela 01 que há chance de nos meses de janeiro, fevereiro, março e dezembro ocorrerem chuvas que ultrapassem $90 \mathrm{~mm}$ mensais, com 30\% de probabilidade de ocorrência. Para os demais meses, eventos dessa magnitude não foram observados. Quando a probabilidade aumenta para 95\%, chuvas superiores a $300 \mathrm{~mm}$ mensais são esperadas, nos referidos meses.

Nos meses de junho, julho e agosto com probabilidade de $95 \%$ de ocorrência as precipitações variam de 6,7 a 15,3 mm mensais, denotando como período crítico de escassez de água. Deve-se propor ações de mitigação para a escassez hídrica objetivando atender a demanda humana, animal e agrícola.

Na Tabela 2 observa-se os níveis de probabilidade de ocorrência (\%) para diferentes totais de precipitação mensal em Bom Jesus do Piauí.

Janeiro, fevereiro, março, novembro e dezembro apresentaram probabilidade de ocorrência superiores a $90 \%$, indicando o período chuvoso, e possível época de plantio e acumulo de água. Agosto seria o mês que não se observou 
precipitação. Junho e julho apresentaram quase $100 \%$ de chance de chuvas abaixo dos $60 \mathrm{~mm}$, setembro apresentou probabilidade de $90 \%$ para precipitação máxima de $25 \mathrm{~mm}$.

Tabela 2. Níveis de probabilidade de ocorrência (\%) para diferentes totais de precipitação mensal em Bom Jesus do Piaui.

\begin{tabular}{|c|c|c|c|c|c|c|c|c|c|c|c|c|}
\hline \multirow{2}{*}{ Precipitação (mm) } & \multicolumn{10}{|c|}{ Níveis de probabilidade de ocorrência (\%) } \\
\cline { 2 - 15 } & jan & fev & mar & abr & mai & jun & jul & ago & set & out & nov & dez \\
\hline 25 & 1 & 2 & 1 & 20 & 72 & 98 & 99 & - & 90 & 35 & 4 & 1 \\
\hline 50 & 5,2 & 9,8 & 4,8 & 37,8 & 85,9 & 99,9 & 100,0 & - & - & 54,2 & 14,8 & 8,4 \\
\hline 60 & 8,1 & 13,8 & 7,7 & 43,9 & 88,9 & 100,0 & 100,0 & - & - & 59,9 & 20,5 & 12,6 \\
\hline 70 & 11,6 & 18,2 & 11,3 & 49,4 & 91,2 & - & - & - & - & 64,7 & 26,6 & 17,5 \\
\hline 80 & 15,6 & 22,9 & 15,4 & 54,5 & 93,0 & - & - & - & - & 69,0 & 32,9 & 22,8 \\
\hline 90 & 19,9 & 27,7 & 19,9 & 59,0 & 94,4 & - & - & - & - & 72,7 & 39,0 & 28,3 \\
\hline 150 & 48 & 55 & 50 & 79 & 98 & - & - & - & - & 87 & 70 & 60 \\
\hline 300 & 90 & 91 & 92 & - & - & - & - & - & - & - & 97 & 95 \\
\hline 500 & 99 & - & - & - & - & - & - & - & - & - & - & - \\
\hline
\end{tabular}

Fonte: Saboya (2022).

Na Tabela 3 observa-se os diferentes períodos de retorno (anos) para as diferentes precipitações mensais (mm) em Bom Jesus do Piaui.

Tabela 3. Períodos de retorno (anos) para diferentes precipitações mensais (mm) em Bom Jesus do Piauí.

\begin{tabular}{|c|c|c|c|c|c|c|c|c|c|c|c|c|}
\hline \multirow{2}{*}{ Precipitação (mm) } & \multicolumn{10}{|c|}{ Período de retorno (anos) } \\
\cline { 2 - 15 } & jan & fev & mar & abr & mai & jun & jul & ago & set & out & nov & dez \\
\hline 25 & 1,0 & 1,0 & 1,0 & 1,2 & 3,6 & 64,1 & 141,0 & - & 10,1 & 1,5 & 1,0 & 1,0 \\
\hline 50 & 1,1 & 1,1 & 1,1 & 1,6 & 7,1 & 989,2 & 3586,2 & - & - & 2,2 & 1,2 & 1,1 \\
\hline 60 & 1,1 & 1,2 & 1,1 & 1,8 & 9,0 & - & 12421,6 & - & - & 2,5 & 1,3 & 1,1 \\
\hline 70 & 1,1 & 1,2 & 1,1 & 2,0 & 11,4 & - & - & - & - & 2,8 & 1,4 & 1,2 \\
\hline 80 & 1,2 & 1,3 & 1,2 & 2,2 & 14,3 & - & - & - & - & 3,2 & 1,5 & 1,3 \\
\hline 90 & 1,2 & 1,4 & 1,2 & 2,4 & 17,8 & - & - & - & - & 3,7 & 1,6 & 1,4 \\
\hline 150 & 1,9 & 2,2 & 2,0 & 4,7 & 62,8 & - & - & - & - & 7,7 & 3,3 & 2,5 \\
\hline 300 & 9,9 & 10,7 & 12,0 & - & - & - & - & - & - & - & 30,3 & 19,5 \\
\hline 500 & 154,5 & - & - & - & - & - & - & - & - & - & - & - \\
\hline
\end{tabular}

Fonte: Saboya (2022). 
No mês de janeiro a possibilidade de precipitações mensais maiores que $300 \mathrm{~mm}$ foi de 9,9 anos, e para precipitações superiores a $500 \mathrm{~mm}$ foi de 154,5 anos, essas informações nos permite desenvolver dimensionamento para obras civis com maior confiabilidade.

Junho, julho e agosto apresentaram períodos de retorno bastante elevados, o que denota a escassez hídrico com grande frequência de ocorrência.

As Tabelas 2 e 3 acima proporciona acesso a informações para saber quais meses que mais possuem chances de secas ou que podem ter alta taxa de precipitação e de quanto em quanto tempo podem ocorrer tais eventos novamente. Determinando-se a disponibilidade hídrica, agricultores, engenheiros, responsáveis por gestão de cidades entre outros, podem fazer suas plantações, recuperações de matas ciliares/reflorestamento ou projetos baseados na probabilidade de ocorrência de chuvas, tendo noção de quanto, obras como barragens ou represas como exemplo, podem suportar, ou se para aquele ano haverá disponibilidade hídrica para determinada cultura agrícola, ou haverá chance de ocorrer uma enchente, podendo prevenir desastres.

\section{Conclusões}

A probabilidade de ocorrência de chuvas no município de Inconfidentes é variável para cada mês, mas segue um padrão no decorrer dos anos. Embora meses como janeiro, fevereiro e outubro, sejam mais críticos com a probabilidade de 4,4\%, 2,2\% e 6,7\%, respectivamente para ocorrência de chuvas com mais de $90 \mathrm{~mm}$ diários e menor incidência nos meses de junho e julho com probabilidade de aproximadamente $0 \%$.

A possibilidade de anomalias na precipitação é real mesmo que com a baixa probabilidade.

Bom Jesus do Piauí possui alta disponibilidade hídrica, com maior volume de chuvas concentrado no período de verão, sendo uma região apta a várias culturas agrícolas.

Determinando a disponibilidade hídrica, a probabilidade de ocorrência de eventos extremos e o período de retorno pode-se fazer um planejamento otimizado, ajudando no desenvolvimento de vários tipos de projetos, trazendo benefícios para a sociedade local.

\section{Referências}

Alvares, C.A.; Stape, J.L.; Sentelhas, P.C.; Gonçalves, J.L.M. \& Sparovek, G. (2014). Köppen's climate classification map for Brazil. Meteorologische Zeitschrift v.22, p.711-728.

Alvarenga, L.A. (2012). Precipitação no sudeste brasileiro e sua relação com a Zona de Convergência do Atlântico Sul. Revista Agrogeoambiental, Minas Gerais, v. 4, n. 2, p.01-07, ago.

Brito, J.H.N.; Feitoza, D.M.A. \& Nascimento, N.S. Eventos extremos de precipitação no juazeiro do Norte. In: 2 Workshop InternacionaL, 2. 2015, Município de Barbalha - CE. Resumo de Congresso. Município de Barbalha - CE: Centec, 2015. p. 1 - 8.

Confalonieri, U.E.C. (2003). Variabilidade climática, vulnerabilidade social e saúde no Brasil. Terra Livre, São Paulo, v. 1, n. 20, p.193-204, jul.

INMET - Instituo Nacional de Meteorologia. (2019). Banco de dados meteorológicos para ensino e pesquisa. <http://www.inmet.gov.br/portal/index.php?r=bdmep/b dmep>.

INMET - Instituto Nacional de Meteorologia. (2019). < http://www.inmet.gov.br/portal/>.

Junqueira Júnior, J.A.; Mello, C.R. \& Alves, G.J. (2015). Eventos extremos de precipitação no Alto Rio Grande, MG: Análise probabilística. Revista Brasileira de Engenharia Agrícola e Ambiental, Campina Grande, v. 19, n. 4, p.301-308, mar.

KÖPPEN, W. (1931). Grundriss der Klimakunde: Outline of climate science. Berlin: Walter de Gruyter. P.388.

Köppen, W.; Geiger, R. Klimate der Erde. (1928). Gotha: Verlag Justus Perthes. Wall-map 150 x $200 \mathrm{~cm}$.

Marengo, J.A. (2009). Mudanças Climáticas e Eventos Extremos no Brasil. Rio de Janeiro: Fbds.

Marengo, J.A. (2008). Água e mudanças climáticas. Estudos Avançados, São Paulo, v. 22, n. 63, p.83-96. 
Research, Society and Development, v.11, n.3, e32411326609, 2022

(CC BY 4.0) | ISSN 2525-3409 | DOI: http://dx.doi.org/10.33448/rsd-v11i3.26609

Medeiros, R.M. (2016). Análise de aspectos climático, socioeconômico e ambiental e seus efeitos na bacia hidrográfica do rio Uruçuí Preto e entorno. Tese de Doutorado em Meteorologia. Universidade Federal de Campina Grande, UFCG, Campina Grande, Brasil. p.173.

Medeiros, R.M. (2016) Estudo agro climatológicos do Estado do Piauí. p. 127.

Mello, C.R.; Viola, M.R. (2013). Mapeamento de chuvas intensas no estado de Minas Gerais. Revista Brasileira de Ciência do Solo, Viçosa, v. 37, n. 1, p.3744 , fev.

Mello, C.R.; Viola, M.R. \& Mello, J.M. (2007). Continuidade espacial de chuvas intensas no Estado de Minas Gerais. Ciência e Agro tecnologia, Lavras, v. 32 , n. 2, p.532-539, fev.

Pereira, A.R.; Angelocci, L.R. \& Sentelhas, P.C. (2007). Meteorologia Agrícola. ESALQ, Departamento de Ciências Exatas. Piracicaba, SP. 192 p.

Pereira, M.W.M.; Balieiro, K.R.C. \& Pinto, L.V.A. (2011). Avaliação da produtividade e adaptabilidade de acessos de amendoim forrageiro para potencial formação/consorciação de pastagens mais sustentáveis no sul de Minas Gerais. In: II Congresso Brasileiro de Gestão Ambiental, 2. 2011, Londrina- Paraná. Resumo de Congresso. Londrina: Ibeas. p. 1 - 6.

Saboya, L.M.F.; Medeiros, R.M.; Holanda, R.M.; França, M.V.; Rolim Neto, F.C. \& Araújo, W.R. (2021). Precipitation probabilities with use of the Gamma Distribution for the municipality of Barbalha-Ce, Brazil. Ijsrm.Human; Vol. 19 (2): 122-134.

Sansigolo, C. A. (2008). Distribuições de extremos de precipitação diária, temperatura máxima e mínima e velocidade do vento em Piracicaba, SP (19172006). Revista Brasileira de Meteorologia, Piracicaba, v. 23, n. 3, p.341-346, mar.

Santos, G.G.; Griebeler, N.P. \& Oliveira, L.F.C. (2009). Chuvas intensas relacionadas à erosão hídrica. Revista Brasileira de Engenharia Agrícola e Ambiental, Campina Grande, Paraíba, v. 14, n. 2, p.115-123, set.

SUDENE - Superintendência de Desenvolvimento do Nordeste. Dados pluviométricos mensais do Nordeste - Paraíba. Recife, (Série Pluviometria, 2). 1990

Weschenfelder, A.B.; Pickbrenner, K.; Pinto, E.J.A. Análise da frequência de ocorrência e a classificação das precipitações diárias máximas anuais na região da laguna dos patos (sub-bacia 87). In: XIX simpósio brasileiro de recursos hídricos. Resumo de Congresso. Maceió: Abril, 2011. p. 01 - 20. 